\title{
Solar-Blind Ultraviolet Upwelling Radiance Diurnal Variation Led by Observation Geometry Factors on Geostationary Attitude Sensor Limb Viewing
}

\author{
Chun-guang Lyu ${ }^{1,2}$, Jia Tian ${ }^{3}$, Wen-bo Yang ${ }^{4,5}$, Qing-jiu Tian ${ }^{1,2, *}$, Ying-hao Lin ${ }^{1,2}$, Zong-ming Liu ${ }^{4,5}$, and \\ Han-mo Zhang 4,5 \\ ${ }^{I}$ International Institute for Earth System Science, Nanjing University, Nanjing, China \\ ${ }^{2}$ Jiangsu Provincial Key Laboratory of Geographic Information Science and Technology, Nanjing University, Nanjing, China \\ ${ }^{3}$ School of Civil and Environment Engineering, Cornell University, Ithaca, New York, USA \\ ${ }^{4}$ Shanghai Institute of Spaceflight Control Technology, Shanghai, China \\ ${ }^{5}$ Shanghai Key Laboratory of Aerospace Intelligent Control Technology, Shanghai, China
}

Received 24 November 2015, revised 6 May 2016, accepted 11 May 2016

\begin{abstract}
In this satellite autonomous navigation feasibility study on geostationary orbit using the ultraviolet (UV) attitude sensor, the solar-blind UV (SBUV) upwelling radiance spatial distributions from the Earth surface points or the limb viewing path are simulated every two hours in all seasons based on the MODTRAN4 model. The target points are arranged from where the sensor can receive the Earth's radiance, including eight limb viewing points in the $0-90 \mathrm{~km}$ altitude range with an interval of 5 $\mathrm{km}$ and nine surface points. A hypothetical UV attitude sensor with $9.6^{\circ}$ field of view is set at a geostationary orbit, $3.6 \times 10^{7} \mathrm{~m}$ away from Equator sea level. The nadir point of which is considered to be located at $102^{\circ} \mathrm{E}$ and the Equator. The results show that SBUV (200 - $280 \mathrm{~nm}$ ) upwelling radiance from the limb viewing points can increase to a maximum and then decrease with the increase in limb points altitude. The average SBUV radiance on the observation plane presents a regular bright crescent or ring, which has the maximum at about $0.433 \mathrm{~W} \mathrm{~m}^{-2} \mathrm{sr}^{-1} \mu \mathrm{m}^{-1}$ at $50-60 \mathrm{~km}$ altitude. The maximum upwelling radiance in the $242-267 \mathrm{~nm}$ spectra has the optimal characteristic considering the contrast and maximum altitude radiance continuity at the limb points.
\end{abstract}

Key words: Passive remote sensing, Optical spectrum analysis, Solar-blind ultraviolet, Limb viewing, MODTRAN4, Geostationary orbit Citation: Lyu, C., J. Tian, W. Yang, Q. Tian, Y. Lin, Z. Liu, and H. Zhang, 2016: Solar-blind ultraviolet upwelling radiance diurnal variation led by observation geometry factors on geostationary attitude sensor limb viewing. Terr. Atmos. Ocean. Sci., 27, 943-953, doi: 10.3319/TAO.2016.05.11.01(AA)

\section{INTRODUCTION}

Autonomous satellite navigation has drawn much attention in recent years. This means that satellite position and velocity determination in real-time without external support assistance is now possible (Xiong et al. 2009; Huang et al. 2010; Ning et al. 2013). Several techniques such as optical sensors, inertial sensors and magnetometers could collect real-time measurement data to realize autonomous satellite navigation (Hablani 2009; Xiong et al. 2013; Wang et al. 2014b). Based on measurement channels, the optical navigation system includes ultraviolet (UV) sensors, visible sensors, and infrared horizon sensors, which are all vision

\footnotetext{
* Corresponding author

E-mail:tianqj@nju.edu.cn
}

systems necessary to observe and recognize the reflected or emitted radiance of space objects (the Earth, the Moon, the Sun, other planets or stars) (Qian et al. 2013). An UV attitude sensor can capture reflected radiance in the solar UV spectrum as well as space contour features when the sensor takes the Earth as an observed target (Qiao et al. 2009). It is crucial and meaningful that the regular Earth UV contour image data can timely provide abundant information with regard to both attitude and position for a satellite. A proper observation mode can largely contribute to hold the regularity and stability of the radiance spatial distribution.

From a new scientific perspective, it is necessary that the Earth's radiance spatial pattern and its all-time variation should be described and evaluated to select a solar-blind UV (SBUV) channel for geostationary orbit (GEO) platforms as 
a discussed observation mode. For historical and technical reasons the former UV attitude sensor was accustomed to choosing the near UV (NUV) spectrum as the measurement channel (Kollmeier et al. 2007; Chahl and Mizutani 2012). Traditional SBUV technology applications often focuses on detecting military tasks on the ground or in the sky using a man-made SBUV emission source (Wang et al. 2014a). The passive SBUV is reasonable and probable for use for Earth image recognition by satellite attitude sensors in that it can effectively avoid the radiance image disturbance caused by complex lower atmospheric composition and surface reflection. On the other hand, a GEO platform is seldom associated with vision imaging by spacecraft attitude sensors (Bak et al. 2012; Uudus et al. 2013). A GEO satellite has the ability to observe a wider field of view than low Earth orbit (LEO) to the benefit of the whole Earth image ( $\mathrm{Li}$ and Sun 2003; Kedar and Arnon 2006). In addition, GEO sensors can lock a constant scene of view by reason that its satellite orbit period coincides with the Earth's rotational period, from which the targets in the field of view can serve as an approximate constant background in time to minimize image uncertainty (Bovensmann et al. 2002; Pahlevan et al. 2013).

It is desired that the SBUV channel and GEO platform merits be combined to discuss Earth contour image recognition by satellite attitude sensors. The potential of this approach can be explored and developed as a new point of interest in image recognition research to provide quantitative data support and references for a prospective Earth attitude sensor. The main objective of this study is to predict and describe Earth radiance spatial pattern in SBUV and its alltime variation on GEO platforms. The solar incident angle greatly determines the SBUV Earth radiance spatial distribution shape so that it, as a primary driving force is discussed with instantaneous Earth image stability and specific measurement channel selection. In order to quantitatively describe and evaluate the all-time Earth radiance spatial pattern, SBUV Earth radiance images on a GEO platform are simulated using the MODTRAN4 model. The variation in SBUV Earth radiance image analysis in a day include: (1) the upwelling radiance features from the Earth limb path in the SBUV spectra range with changing altitudes; (2) the radiance bright region shape and spatial distribution on the sensor observation plane with the temporal variation in a day; and (3) the spectrum range option based on the contrast and altitude continuity of maximum radiance at the Earth limb sample points considering the all-time variation in simulated radiance image data.

\section{METHOD}

\subsection{Assumption}

When a wavelength is shorter than $300 \mathrm{~nm}$, solar radiation is strongly absorbed by some trace gas like ozone, so that it barely arrives some area such as the Earth's surface and even the troposphere. As a result, this space is called the UV Solar-blind region. The longer wavelength solar radiation $(>300 \mathrm{~nm})$ is able to get through the stratosphere ozone layer, but will be affected by complicated scatters, absorbers and reflectors in the lower atmosphere such as aerosols, clouds, and the surface so that surface reflectance and the tropospheric condition have great variability on a global scale and will significantly determined the Earth disk radiance distribution (Bhartia et al. 1993). According to the radiance contribution function (Bhartia et al. 1996), the SBUV (roughly the equivalent of middle UV, $200-300 \mathrm{~nm}$ ) radiance is dominated by stratospheric Rayleigh scattering above the stratosphere ozone layer. The stratospheric condition above the ozone layer is far more steady and pure than that in the lower troposphere near the Earth's surface, which is also one reason to choose the SBUV band to retrieve radiance distribution stability features for sensor attitude. Only one the Earth limb annulus can then be locked by a GEO sensor, so that the certainty and predictability of this limb atmosphere is greatly increased. An assumed broad detection band and wide instantaneous field of view to recognize the Earth's image could also largely weaken sensor signal sensibility for the atmospheric conditions and surface.

Therefore, according to the above, SBUV upwelling radiance simulation on the two assumptions that: (1) the signal spatial distribution detected by the SBUV sensor is determined mainly by stratospheric conditions above the ozone layer and not sensitive to the diurnal variation in the Earth's atmospheric conditions in the time range of a day; (2) the surface reflectance contribution could be neglected in the SBUV upwelling radiance at the sensor entrance.

Based on the above assumptions, solar incident angle and observation path have been the primary variable factors considered in simulating radiance at the sensor entrance. The core problem is the variation in spectra radiance with observation geometry as well as the radiance stability features assessment from each Earth limb path. Except for Earth limb points, some surface points are considered simulated as background values for stand out distribution features of the Earth limb radiance.

\subsection{Simulation Strategy}

\subsubsection{Estimation of Earth Radiance Based on MODTRAN4}

The realistic Earth disk upwelling radiance should be obtained by taking accurate scattering and absorption estimation in the terrestrial atmosphere and the Earth surface reflection contribution into account at high spectral resolution over the SBUV spectra (Berk et al. 1999; Börner et al. 2001). The Moderate spectral resolution atmospheric transmittance algorithm and computer model (MODTRAN) is well suited to this task as it is a computationally rigorous radiation transfer algorithm used to model atmospheric spectral absorption, 
transmission, emission, and scattering characteristics.

The $4^{\text {th }}$ version, MODTRAN4, is an atmospheric model developed by the US Air Force Research Laboratory over the past 25 years. MODTRAN4 can calculate atmospheric transmittance or radiance over the $0-50000 \mathrm{~cm}^{-1}$ frequency range or wavelengths greater than $200 \mathrm{~nm}$ at moderate spectral resolution, primarily $2 \mathrm{~cm}^{-1}$ from a specified path through the atmosphere. It provides many ways to define the calculation geometry considering effects caused by atmospheric refraction and the Earth's curvature. The atmosphere can be treated as a stack of up to 34 atmospheric layers, from 0 - $100 \mathrm{~km}$ altitude, and freely define the physical parameters in each layer, ranging from pressure, temperature, number density of gasses to molecular absorption and extinction coefficients. As the path travels through each layer in the model, MODTRAN4 has the capability to calculate single and multiple scattering (Rayleigh and Mie caused by gases, aerosols, clouds, fogs, and rain) relying on the DISORT (discrete ordinates radiative transfer) algorithm (Smith et al. 2013) and sum the fluxes over the path and wavelength band. The MODTRAN4 model can simulate the SBUV radiance at the GEO sensor entrance pupil, including the full path from the Earth limb or surface through the terrestrial atmosphere, as illustrated in Fig. 1a.

\subsubsection{Arrangement of Simulated Sampling Points and Timing}

All target points to be simulated could be projected vertically at the Earth observation plane, as shown in Fig. 1b. On the Earth disk the equator and the meridian where the nadir is located are converted into horizontal and vertical coordinates. As the intersection between the two coordinates, the nadir point serves as the origin for the projected observation

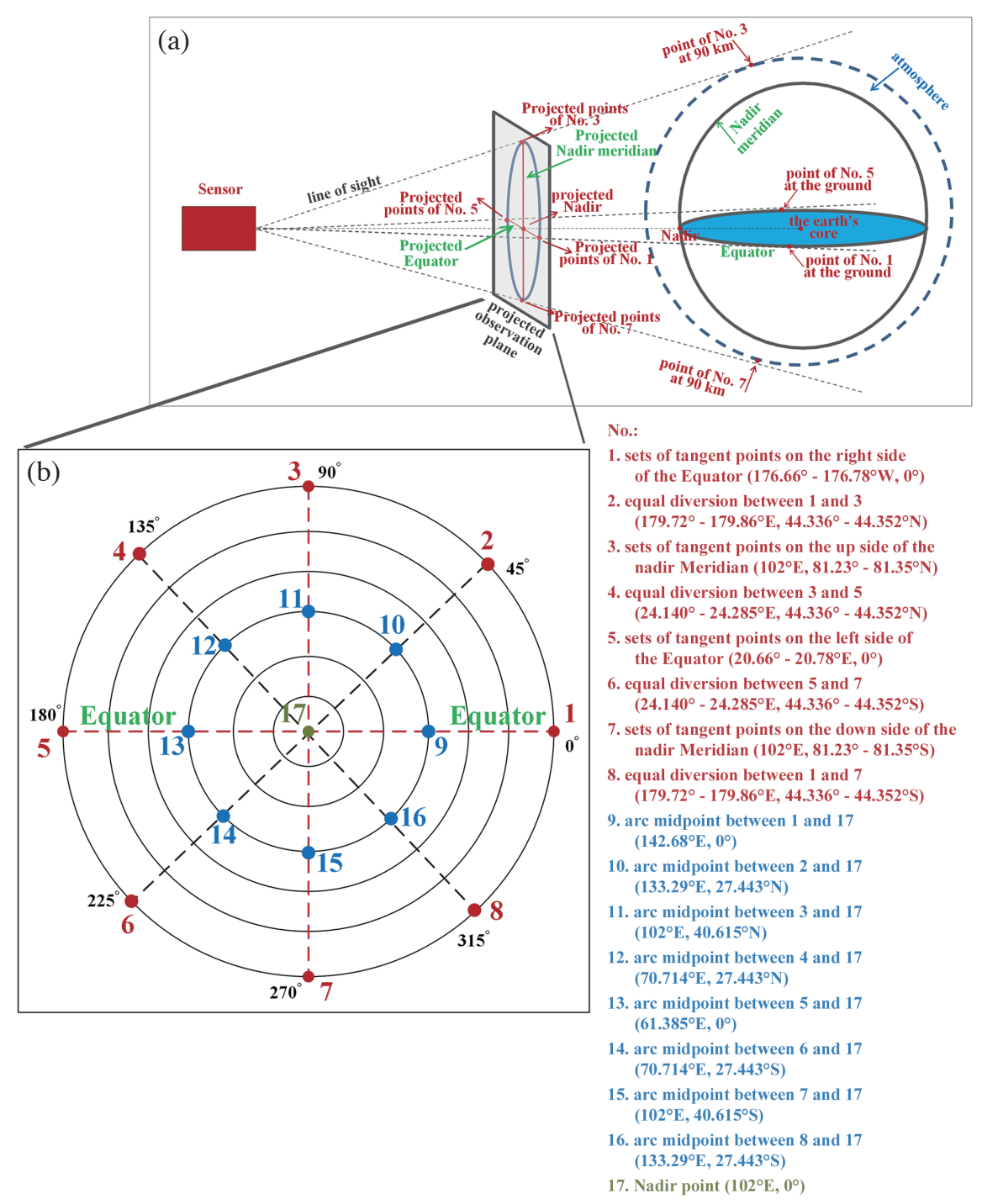

Fig. 1. (a) Geometrical relationship among the sensor, the Earth and the observation plane; (b) simulated sampling points on the observation plane and their serial number. (Color online only) 
plane, from which the simulated sampling points are regularly arranged at eight equiangular directions. Among the sampling points, the Earth limb tangent points are all at the edge of the Earth disk plane, each of which is actually the sets of simulated points at different altitudes and are refined into 20 equal intervals from the ground to $100 \mathrm{~km}$ in the Earth atmosphere. Beside the sets of limb points at 8 directions of nadir, 8 additional equal diversion points are considered between each limb point and nadir to capture the Earth radiance spatial pattern for a time. The detailed simulated sampling point arrangement is as shown in Fig. 1.

On the GEO platform the satellite is synchronous with the Earth's rotation. Therefore, the sun right ascension could be in continuous shift in an Earth rotational period. In combination with the observation angle results in the absorption and scattering effect difference in a specific path, serving as the dominant factor to the Earth radiance variation at every time point in a day in the SBUV. The equinox days and solstice days can be chosen as four optimal dates to represent the extreme value $\left(23^{\circ} 26^{\prime}\right)$ and median $\left(0^{\circ}\right)$ of the sun declination, so that the seasonal variation in radiance can be expressed. Every 2 hours was set as the time interval to obtain the radiance of all sampling points on the Earth disk corresponding to every $30^{\circ}$ that the Sun moves round the Earth. In our research, local time in nadir is taken as the reference time and its zero a.m. is considered the simulated initial time point convenient to the following description. The sun-observer angle (SOA), ranging from $-180^{\circ}$ to $180^{\circ}$, is closely related to the reference time defined as the difference in value of the longitude between the Sun and observer. When the reference time is from 0 a.m. to 12 noon, accordingly the range of SOA shifts from $-180^{\circ}$ to $0^{\circ}$. Naturally, while the local time is from 12 noon to 12 p.m., its range changes from $0^{\circ}$ to $180^{\circ}$.

\subsection{Detailed Flow}

In summary, MODTRAN4 can be seen as a black box, providing inputs port for several kinds of parameters and options (see the Table 1) related to the Earth radiance simulation on an assumed satellite orbit in a special time and date. It is controlled to output simulated spectral data for each sampling point until all time and date processes are completed. The spectrum integral or spatial interpolation is executed according to the following analysis and discussion requirements. The detailed flow is presented in Fig. 2.

\section{RESULTS AND ANALYSIS}

\subsection{The Radiance Features in 200 - $300 \mathrm{~nm}$ at the Earth Limb}

The variation in right sun ascension and declination determines the absolute radiance value at any point on the Earth disk, however the difference in radiance along the lamb point

Table 1. Key input parameters and options for MODTRAN4.

\begin{tabular}{cc}
\hline Parameter/option & Value \\
\hline Model Atmosphere & $390 \mathrm{ppmv}$ \\
$\mathrm{CO}_{2}$ Mixing Ratio & Rural/Maritime \\
Aerosol Model & $100 \mathrm{~km}$ \\
Observer Height & every $5 \mathrm{~km}$ interval from $0-100 \mathrm{~km}$ \\
Final Height & $93.186 \sim 180 \mathrm{deg}$. \\
Zenith Angle & $200 \sim 300 \mathrm{~nm}$ \\
Spectral Range & $5 \mathrm{~cm}{ }^{-1}$ \\
FWHM of Slit Function & Gaussian \\
Slit Function Type & 8 Streams \\
Number of Streams & yes \\
DISORT Azimuthal Dependence & Lambertian surface \\
Surface Albedo & Corrected Kurucz database \\
Solar Database Option & MIE Generated \\
Aerosol Phase Function & 80 \\
Day of Year & 102 deg. E, equator \\
longitude and latitude of the Nadir & $-81.35 \sim 81.35$ deg. \\
Target latitude & $176.66 \sim 339.34$ deg. \\
Target longitude & spring equinox, summer solstice, autumnal equinox, and winter solstice \\
Greenwich Time & $0 \sim 315$ deg. \\
Date &
\end{tabular}




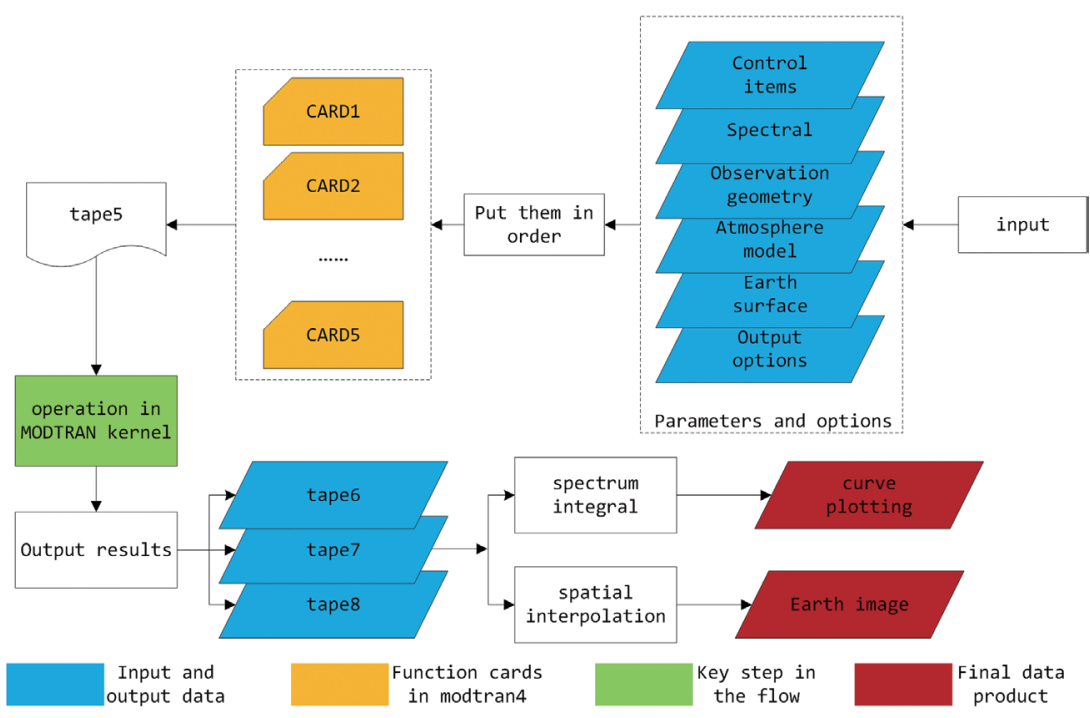

Fig. 2. The detailed flow to simulate and output data. (Color online only)

attitudes practically depend only on itself. Hence, the limb UV upwelling radiance from point No.1 can be taken as an example to describe the characteristics due to the similarity in the shape and trend of the radiance from the limb viewing path in the UV spectrum. Figure 3 shows the SBUV radiance curves in the range from 0 to around $3.5 \mathrm{~W} \mathrm{~m}^{-2} \mathrm{sr}^{-1} \mu \mathrm{m}^{-1}$. The radiance maximum in the $200-300 \mathrm{~nm}$ is at the 50 or $60 \mathrm{~km}$ altitude. The radiance in the $288-300 \mathrm{~nm}$ is significantly higher than other ranges in the $200-300 \mathrm{~nm}$ spectra, as shown in Fig. 3. The radiance caused by scattering is weak at the upper atmosphere such as $90 \mathrm{~km}$ altitude because of the fairly low density of molecules.

\subsection{Spatial Distribution of Diurnal SBUV Radiance from the Earth Limb and Surface}

The radiance from the limb path at lower tangent altitude is higher than that transferred from the Earth's surface or the higher tangent altitude (less than $90 \mathrm{~km}$ ) of the limb. Therefore, the radiance bright side would be formed due to the relatively high value area between the Earth's surface and upper atmosphere, and it exists in the Earth limb at any time in a day. The high value of the radiance is distributed mainly in the altitude range between 40 and $60 \mathrm{~km}$. On the observation plane, the overall intensity and radiance spatial distribution is greatly affected by the combined action of SOA (time, solar hour angle or solar right ascension) and declination angle (date or obliquity of the ecliptic). The change in SOA will bring about radiance lateralization at the left and right sides of the Earth disk. Meanwhile, the alteration in declination angle could result in unbalanced radiation energy detected by the sensor at the up and down sides of the Earth disk in the UV spectra. The vernal equinox day could be selected as a reasonable date to draw the SBUV radiance distribution on the Earth disk (as seen in Fig. 4), that is beneficial in emphasizing the radiance at the left and right sides of the Earth disk with time. By comparison, at several times, it is expressed that the up and down features of the radiance spatial distribution rely on declination angle alteration (as seen in Fig. 5).

Figure 4 shows the spatial distribution in the integral SBUV spectra radiance on the Earth disk with SOA every $30^{\circ}$ ( 2 hours). In a certain range of the limb or surface opposite to the direct sunlight point, the upwelling radiance is zero by reason of the block by the Earth. The radiance concentrates on the right side of the Earth disk (see Figs. 4a - f). While the SOA are between $0^{\circ}$ and $180^{\circ}$, the high values shift to the left side of the disk (see Figs. $4 \mathrm{~g}-\mathrm{k}$ ). When the SOA is $-180^{\circ}$, that means, from the observation directions to nadir, the sun is directly behind the Earth, the radiance from the Earth's surface is zero, and the limb path is very small. On the other hand, bright edge of radiance exists simultaneously on left and right sides of the Earth disk at $180^{\circ}$ SOA (12:00 p.m. in Nadir time) (see Fig. 4f). According to Figs. $4 \mathrm{a}$ and 1 , the radiance bright side of the limb could grow up at the right side of the observation plane with the SOA changing from $-180^{\circ}$ to $-150^{\circ}$. The following trend is that the bright side gradually darkens at the right side with the angle variation from $-150^{\circ}$ to $-90^{\circ}$ (see Figs. $4 \mathrm{~b}$ and c) and then brightens with the $-90^{\circ}$ to $0^{\circ}$ (see Figs. $4 \mathrm{~d}$ and e). While, the bilateral bright side convert to the unilateral bright side on the left side of the disk, and it has an inverse trend as the radiance variation of the right side with the change in angle from $0^{\circ}$ to $180^{\circ}$ (Figs. $4 \mathrm{~g}-\mathrm{k}$ ).

Figure 5 shows the integral SBUV spectra radiance on the Earth disk at several times on the summer solstice, equinox day and winter solstice, respectively. The solar declination determines the degree that sunlit point of the 
sun deviated from the observation plane horizontal axis. On the summer solstice the sunlit point located at the Northern Hemisphere and the solar declination to be maximized result in high density radiant energy on the upper Earth disk (Figs. 5a-d). With the decrease in solar declination angle on (spring or autumn) equinox day, the terrestrial equator can go across the sunlit point and the radiance spatial distribution is then axial symmetric about the Earth disc horizontal axis (Figs. 5e - h). When the sunlit point shifts to the Southern Hemisphere, the radiant energy is gradually transferred to the Earth disk down side. On the winter solstice the solar declination to be maximized and the radiance spatial distribution is shown in Figs. 5i - 1. At specific times and dates the observation geometry, atmospheric density latitudinal discrepancies, etc., can serve as factors to slightly reshape some part of the Earth disk bright side.

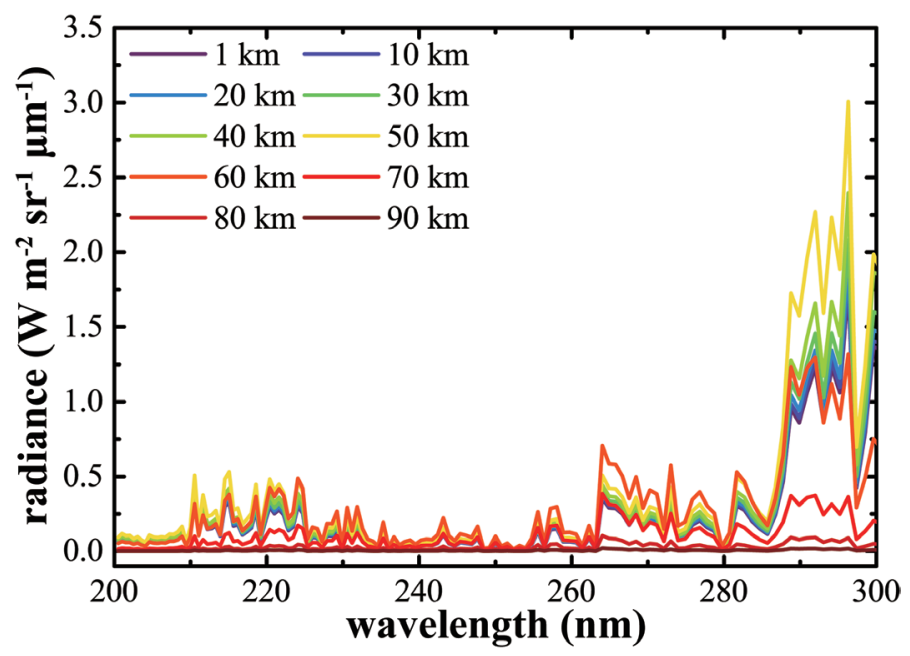

Fig. 3. Limb UV upwelling radiance from the No. 1 points at different altitudes in the $200-300 \mathrm{~nm}$ spectra at 12:00 p.m., vernal equinox day in nadir.

(a)

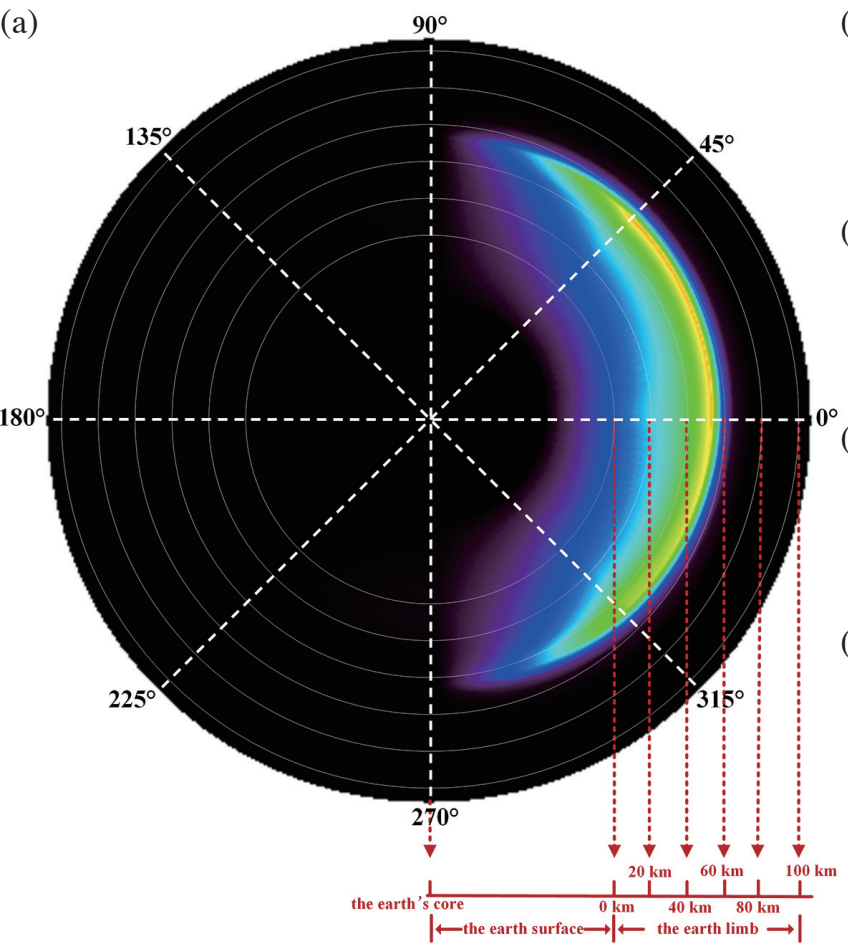

(b)

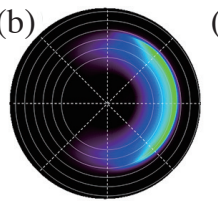

(e)
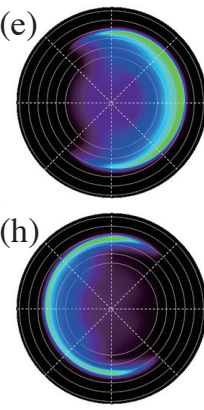

(k)
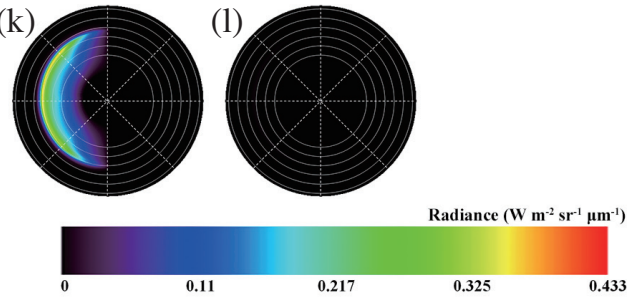

(c)

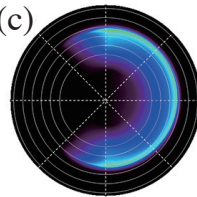

(f)
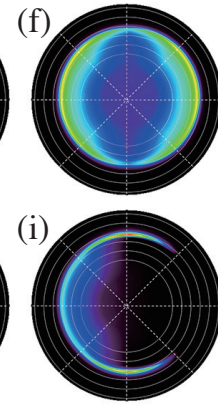

(1)

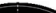

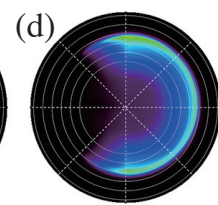

(g)

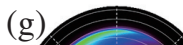

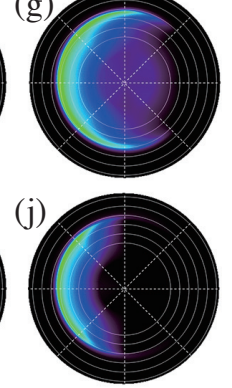

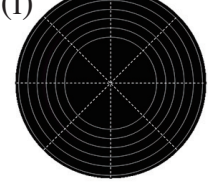
. 

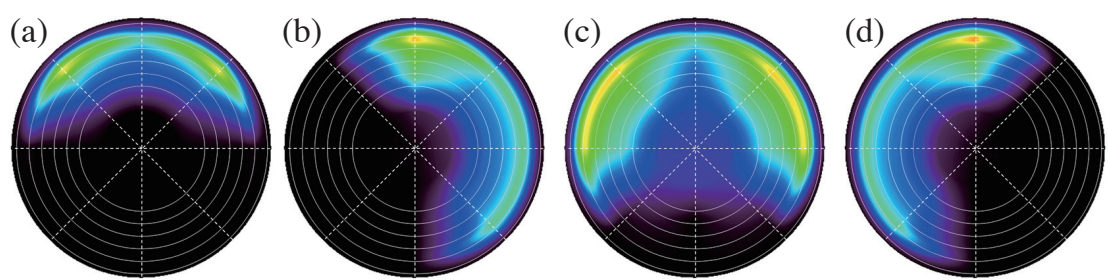

Radiance $\left(\mathrm{W} \mathrm{\mathbf {m } ^ { - 2 }} \mathbf{s r}^{-1} \mu \mathrm{m}^{-1}\right)$
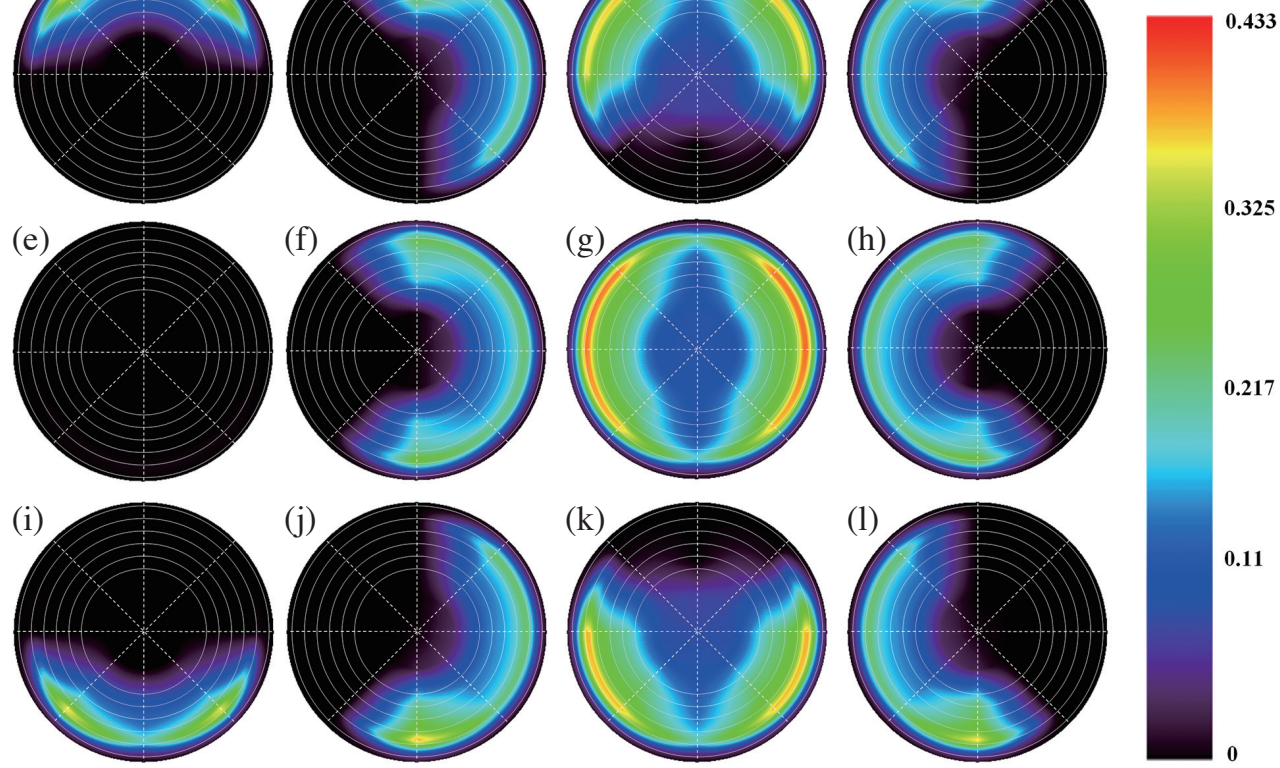

0.11

Fig. 5. The SBUV radiance distribution from the Earth's surface and limb viewing path in Earth disk with the changes of sun declination angles. summer solstice: (a) at 0:00 a.m., (b) at 6:00 a.m., (c) at 12:00 p.m., (d) at 6:00 p.m.; equinox day: (e) at 0:00 a.m., (f) at 6:00 a.m., (g) at 12:00 p.m., (h) at 6:00 p.m.; winter solstice: (i) at 0:00 a.m., (j) at 6:00 a.m., (k) at 12:00 p.m., and (l) at 6:00 p.m. in Nadir time. (Color online only)

\subsection{Analysis of UV Radiance Stability and Differentiation at the Earth Limb}

Figure 6 shows the maximum SBUV radiance altitudes from the limb path. Most of them fluctuate in the altitudes from $40-80 \mathrm{~km}$ with the change in SBUV Spectra. In order to analyze the tangent altitude stability at where the maximum upwelling radiance are located on the bright side according to Fig. 6, the derivative of all altitudes are taken with respect to the wavelength used to indicate the rate and a stability index is defined as follows [Eq. (1)]:

$\operatorname{index}(\lambda)=\frac{1}{N \cdot T \cdot D} \sum_{i=0}^{d} \sum_{j=0}^{t} \sum_{k=1}^{n}\left|\frac{\partial H(i, j, k, \lambda)}{\partial \lambda}\right|$

where, $H$ is the tangent altitude at where the maximum upwelling radiance is located; $\lambda$ is the wavelength; $i$ is the serial number of dates; $D$ is the number of dates; $j$ is the timing; and $T$ is the simulation time number; $k$ is the serial number for the limb points; $N$ is the number of points.

According to Eq. (1), if the altitude with $\lambda$ does not change at each limb point and every observed time in a day, the stability index is also zero or it is a positive value. Through calculation in every nanometer of the UV spectrum, the spectrums with zero value of the stability index only include 200.5 - 205.5, 218.5 - 221.2, 228.5 - 232.1, and $242.0-267.0 \mathrm{~nm}$ (see Fig. 7). Considering the requirement for broad spectra in practical application, $242.0-267.0 \mathrm{~nm}$ should be a suitable spectrum range.

The relative differences in the upwelling radiance should be considered an indicator for the point coordinates recognition. There are ratio definitions such as the Weber contrast and Michelson contrast to represent the contrast between the object and background or other objects. The maximum upwelling radiance at altitudes of a limb viewing point are considered as the compared quantity, and the radiance contrast of this limb point at some altitude can be defined as:

$$
\begin{aligned}
& C_{k}\left(\theta, \varphi, \theta^{\prime}, \varphi^{\prime}, \lambda\right)= \\
& \frac{L_{H}\left(\theta, \varphi, \theta^{\prime}, \varphi^{\prime}, \lambda\right)-L_{k}\left(\theta, \varphi, \theta^{\prime}, \varphi^{\prime}, \lambda\right)}{L_{H}\left(\theta, \varphi, \theta^{\prime}, \varphi^{\prime}, \lambda\right)}
\end{aligned}
$$

where, $C_{k}$ is the radiance contrast between radiance maximum at this point and the radiance at an altitude for this point; $\theta$ and $\varphi$ are the solar zenith angle and solar azimuth respectively; $\theta^{\prime}$ and $\varphi^{\prime}$ are the LOS zenith angle and LOS azimuth angle; $\lambda$ is the wavelength; $L_{H}$ is the upwelling radiance maximum at altitudes of a point; and $L_{i}$ is the upwelling radiance at $k \mathrm{~km}$ altitude of a point.

The radiance contrast of a limb viewing point should consider every contrast $C$ of the maximum of the radiance with other radiance at altitudes of this point. The altitude difference is also considered as a parameter in the limb viewing point differentiation. The differentiation based on distance-weighted are defined as: 
$D W A C=\frac{1}{N \cdot D} \sum_{i}^{d} \sum_{k}^{h} C_{k} \cdot|H-k|^{-1}(k \neq H)$

where, $D W A C$ is the distance weighted average contrast corresponding to a limb viewing point set; $N$ is the number of altitude intervals; $D$ is the number of dates; $C_{k}$ is the radiance contrast between the radiance maximum and radiance at an altitude; $H$ is the altitude that the radiance maximum appeared; $i$ is the serial number of dates; $k$ is the altitude that non-maximum of radiance appeared; and $h$ is the maximum of altitude.

Figures $8 \mathrm{a}-1$ show the distance weighted average contrast $(D W A C)$ in the UV spectrum. When the SOA is zero (at the 0:00 a.m. in the Nadir time), the DWAC curve has larger fluctuation in the SBUV spectra and the variation in $D W A C$ value among the limb points has no obvious correlation (Fig. 8a), which may be caused by the complicated multiple scattering in the radiative transfer path by the special sun-observer-limb geometry and the influence of blocking by the Earth. At all times except 0:00 a.m., the DWAC of points (nonzero value) at the Equator can keep more stable threshold than that near the Antarctic and Arctic regions in SBUV spectra. Meanwhile, the DWAC curve of each lamb point has relatively high value in the SBUV spectra from $238-267 \mathrm{~nm}$ except for parts of the curves in Fig. 8a.

Since the radiance condition may be useful to some applications such as satellite autonomous navigation, it is easy and important to recognize the limb point position with high brightness and contrast in the sensor observation plane. The relationship between $D W A C$ and the maximum radiance of each limb point on the bright side is illustrated in the optimal spectra range $(242.0-267.0 \mathrm{~nm})$ as the scatter plot (Fig. 9). Figure 9 suggests that it is not possible for the average spectral radiance from a limb point to have higher value and
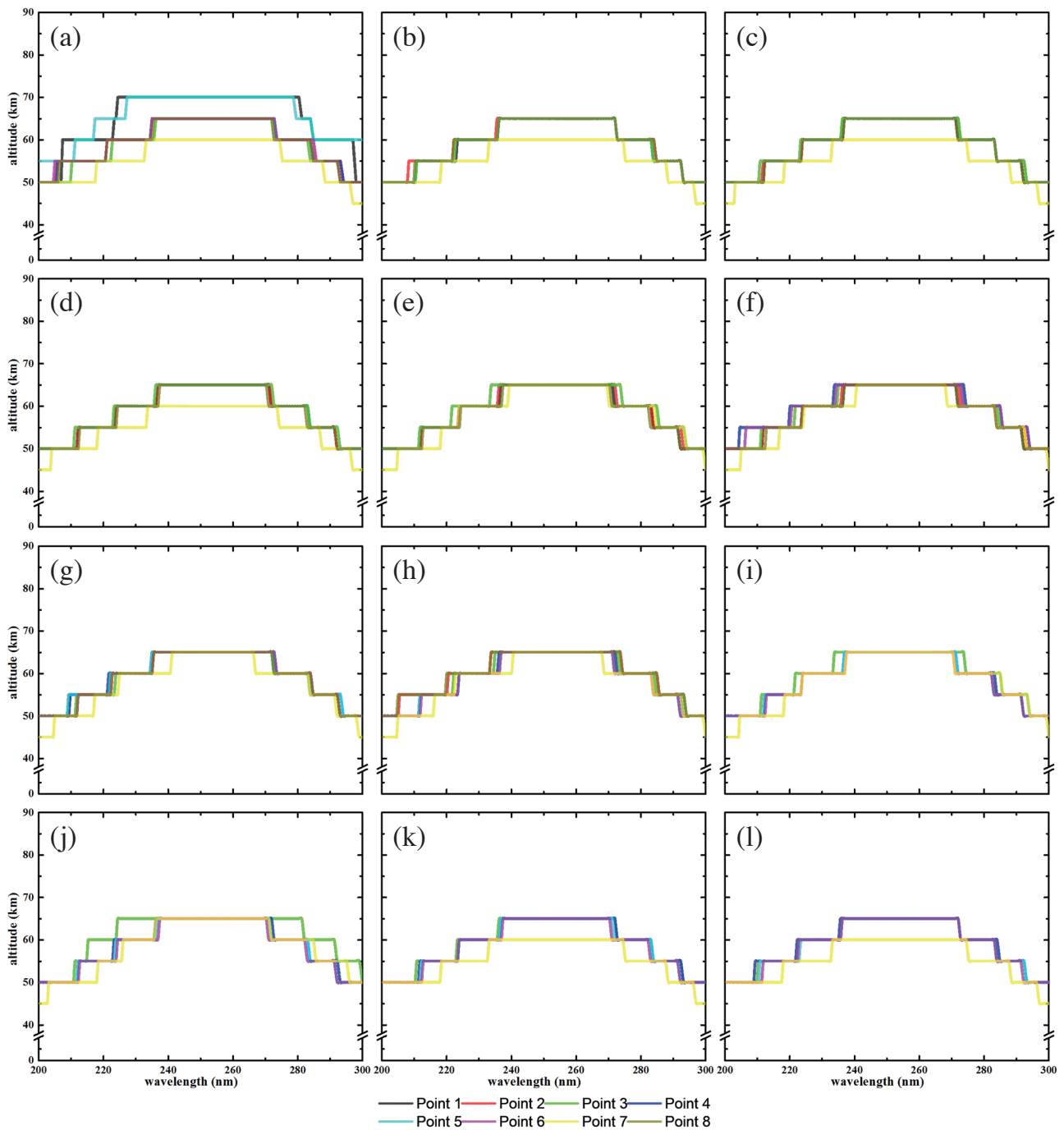

Fig. 6. The altitudes of the maximum upwelling radiance appearing at limb points in the observation plane: (a) at 2:00 a.m., (b) at 4:00 a.m., (c) at 6:00 a.m., (d) at 8:00 a.m., (e) at 10:00 a.m., (f) at 12:00 p.m., (g) at 2:00 p.m., (h) at 4:00 p.m., (i) at 6:00 p.m., (j) at 8:00 p.m., (k) at 10:00 p.m., and (1) at 0:00 a.m. 


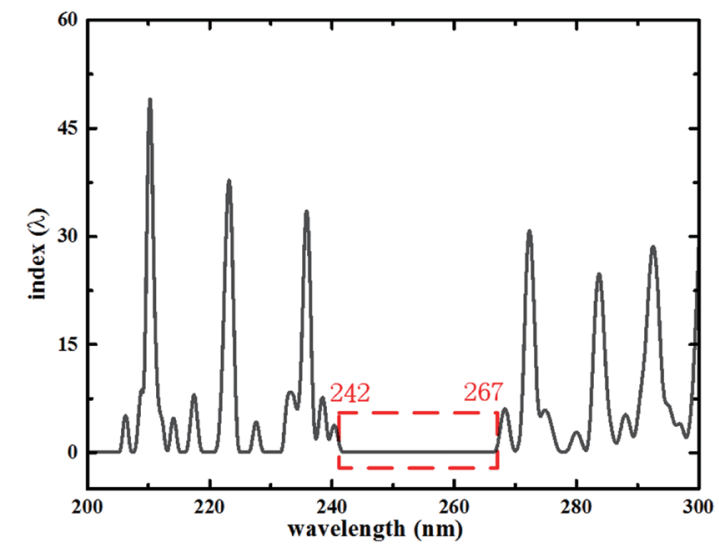

Fig. 7. The stability index in the $200-300 \mathrm{~nm}$ spectra. (Color online only)
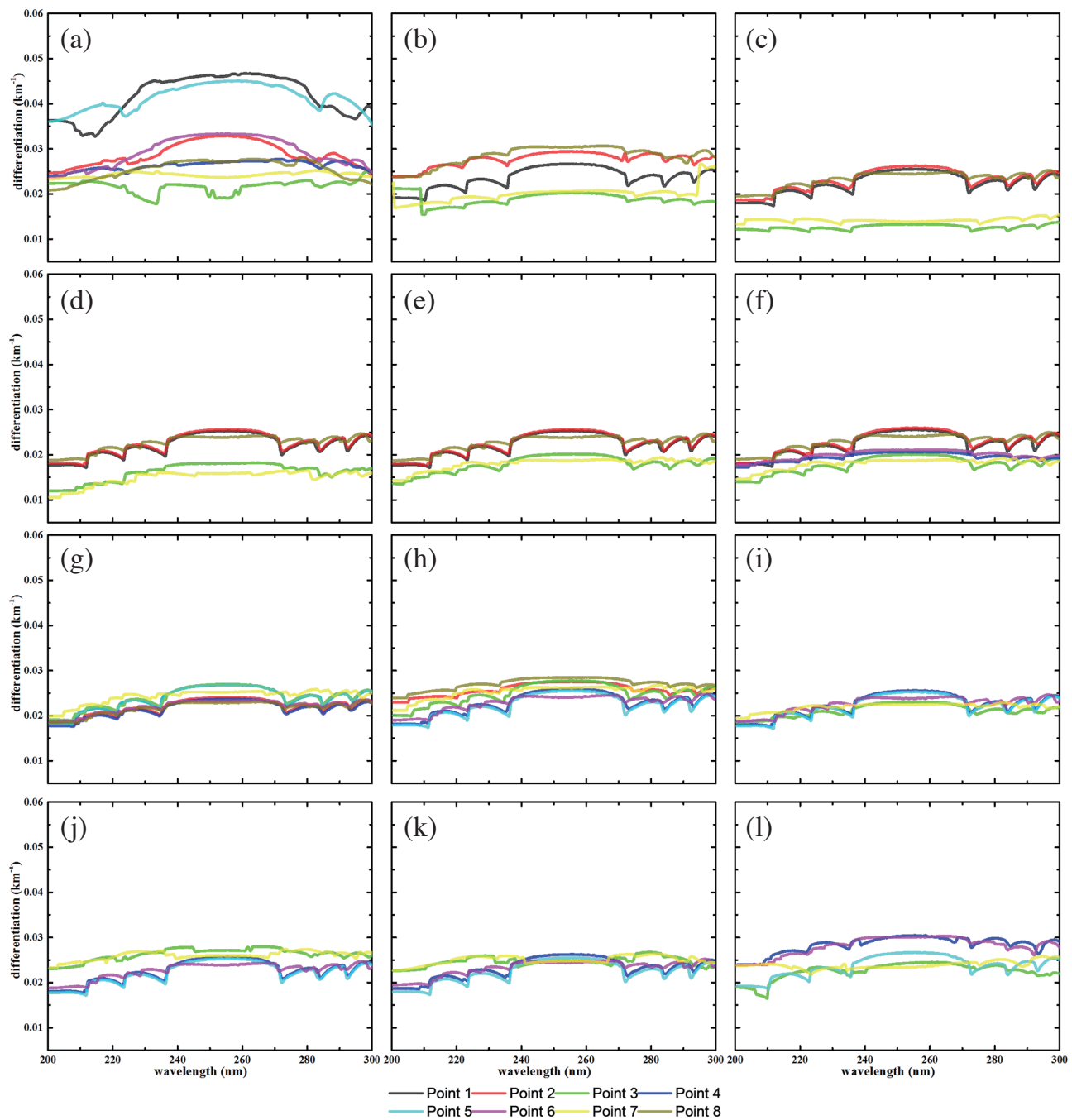

Fig. 8. The DWAC of radiance at altitudes from each limb path in the observation plane: (a) at 2:00 a.m., (b) at 4:00 a.m., (c) at 6:00 a.m., (d) at 8:00 a.m., (e) at 10:00 a.m., (f) at 12:00 p.m., (g) at 2:00 p.m., (h) at 4:00 p.m., (i) at 6:00 p.m., (j) at 8:00 p.m., (k) at 10:00 p.m., and (l) at 0:00 a.m. 


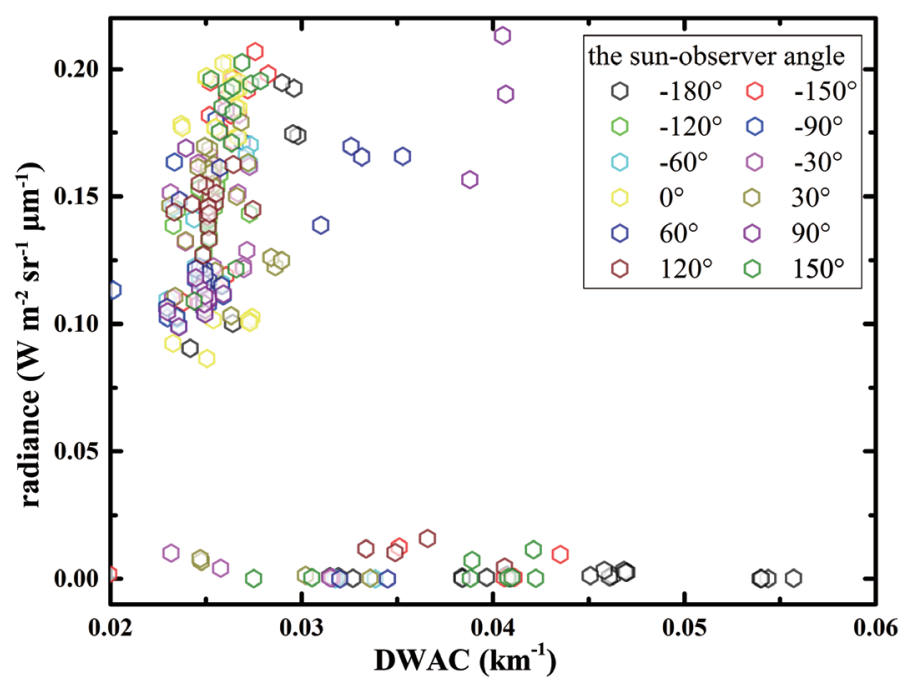

Fig. 9. The correlation between DWAC and average spectral radiance of the altitude that the maximum appeared at each limb points of the bright side with the change of the sun-observer angle in the $242-267 \mathrm{~nm}$.

contrast at the same time. The smaller the SOA, or the limb points location near the Poles always tend to the upwelling radiance having large contrast and small brightness, and the large angle or the points location near the Equator lead to the upwelling radiance holding smaller contrast and larger absolute value.

\section{CONCLUSIONS}

The synthetic radiance into the GEO sensor entrance can be simulated using the MODTRAN4 model considering the Earth surface points and limb points on the observation plane. The geocentric angle between the Sun and observer is a major factor to be discussed. The equinox and solstice days are considered the simulation dates, so that the annual variation in radiance caused by sun declination angle can be involved.

According to the distinction in the SBUV radiance, the spatial distributions of the radiance are illustrated respectively on the Earth disk in SBUV spectral ranges. Due to the lower radiance from the Earth's surface and the upper atmosphere, a relatively high value area exists in a certain limb range on the Earth disk. The high radiance value area could shape a bright side on the left or right side of the observation plane at any time in the simulation day and the bright annulus could be shaped near the $0^{\circ}$ of SOA. The bright side at the right or left of the observation plane alternates light and dark many times with the changes in SOA.

The spectral signatures of the bright side are discussed based on the diurnal variations in the upwelling radiance from the limb path. The two important statistical indexes, the stability index and DWAC are defined to describe the limb tangent altitude continuity and contrast conditions of the maximum radiance, respectively in the UV spectra. The
$242-267 \mathrm{~nm}$ is confirmed as the optimal spectral range based on the stability index and spectral width. In this spectral range most lamb points have higher contrast based on the DWAC. The relationship between the absolute value and contrast in the radiance indicate that there is no such limb point that has both higher radiance and contrast. Therefore, the two factors above should be taken into account in the sensor feasibility design used for the limb point recognition. In the future, sensitivity research on the sensors would be expected based on the diurnal or annual variation in radiance.

Acknowledgements The authors thank Wen-Feng Zhan for their advice on the paper. This work was supported by the Natural Science and Technology Major Project (Grant No. 30-Y20A29-9003-15/17 and 03-Y20A04-9001-15/16 in China), and Specialized Research Fund for the Doctoral Program of Higher Education (Grant No. 20100091110012 in China).

\section{REFERENCES}

Bak, J., J. H. Kim, R. J. D. Spurr, X. Liu, and M. J. Newchurch, 2012: Sensitivity study of ozone retrieval from UV measurements on geostationary platforms. Remote Sens. Environ., 118, 309-319, doi: 10.1016/j. rse.2011.11.010. [Link]

Berk, A., G. P. Anderson, L. S. Bernstein, P. K. Acharya, H. Dothe, M. W. Matthew, S. M. Adler-Golden, J. H. Chetwynd Jr., S. C. Richtsmeier, B. Pukall, C. L. Allred, L. S. Jeong, and M. L. Hoke, 1999: MODTRAN4 radiative transfer modeling for atmospheric correction. Proc. SPIE 3756, Optical Spectroscopic Techniques and Instrumentation for Atmospheric and Space Research III, 348-353, doi: 10.1117/12.366388. [Link] 
Bhartia, P. K., J. Herman, R. D. Mcpeters, and O. Torres, 1993: Effect of Mount Pinatubo aerosols on total ozone measurements from backscatter ultraviolet (BUV) experiments. J. Geophys. Res., 98, 18547-18554, doi: 10.1029/93JD01739. [Link]

Bhartia, P. K., R. D. McPeters, C. L. Mateer, L. E. Flynn, and C. Wellemeyer, 1996: Algorithm for the estimation of vertical ozone profiles from the backscattered ultraviolet technique. J. Geophys. Res., 101, 1879318806, doi: 10.1029/96JD01165. [Link]

Börner, A., L. Wiest, P. Keller, R. Reulke, R. Richter, M. Schaepman, and D. Schläpfer, 2001: SENSOR: A tool for the simulation of hyperspectral remote sensing systems. ISPRS J. Photogram. Rem. Sens., 55, 299-312, doi: 10.1016/S0924-2716(01)00022-3. [Link]

Bovensmann, H., S. Noël, P. Monks, A. P. H. Goede, and J. P. Burrows, 2002: The geostationary scanning imaging absorption spectrometer (GEOSCIA) mission: Requirements and capabilities. Adv. Space Res., 29, 18491859, doi: 10.1016/S0273-1177(02)00104-7. [Link]

Chahl, J. and A. Mizutani, 2012: Biomimetic attitude and orientation sensors. IEEE Sensor. J., 12, 289-297, doi: 10.1109/JSEN.2010.2078806. [Link]

Hablani, H. B., 2009: Autonomous inertial relative navigation with sight-line-stabilized sensors for spacecraft rendezvous. J. Guid. Contr. Dynam., 32, 172-183, doi: 10.2514/1.36559. [Link]

Huang, L., B. Liang, T. Zhang, and W. Xu, 2010: An autonomous navigation method for GEO satellites using X-ray pulsars. $20103^{\text {rd }}$ International Symposium on Systems and Control in Aeronautics and Astronautics (ISSCAA), Harbin, 529-534, doi: 10.1109/ISSCAA.2010.5633073. [Link]

Kedar, D. and S. Arnon, 2006: Non-line-of-sight optical wireless sensor network operating in multiscattering channel. Appl. Optics, 45, 8454-8461, doi: 10.1364/ AO.45.008454. [Link]

Kollmeier, T., F. Röben, W. Schenck, and R. Möller, 2007: Spectral contrasts for landmark navigation. J. Opt. Soc. Am., 24, 1-10, doi: 10.1364/JOSAA.24.000001. [Link]

Li, L. and H. Sun, 2003: A method of astronomical autonomous orbit and attitude determinations for satellites. Chin. Astron. Astrophys., 27, 481-489, doi: 10.1016/ S0275-1062(03)90072-5. [Link]

Ning, X., L. Wang, X. Bai, and J. Fang, 2013: Autonomous satellite navigation using starlight refraction angle measurements. Adv. Space Res., 51, 1761-1772, doi: 10.1016/j.asr.2012.12.008. [Link]

Pahlevan, N., Z. Lee, C. Hu, and J. R. Schott, 2013: Analyzing radiometric requirements for diurnal observations of coastal/oceanic waters from geostationary orbits. Proc. SPIE 8724, Ocean Sensing and Monitoring V, 87240K, doi: 10.1117/12.2016279. [Link]

Qian, Y., C. Li, W. Jing, I. Hwang, and J. Wei, 2013: SunEarth-Moon autonomous orbit determination for quasiperiodic orbit about the translunar libration point and its observability analysis. Aero. Sci. Tech., 28, 289296, doi: 10.1016/j.ast.2012.11.009. [Link]

Qiao, L., J. Liu, G. Zheng, and Z. Xiong, 2009: Augmentation of XNAV system to an ultraviolet sensor-based satellite navigation system. IEEE J. Sel. Top. Signal Process., 3, 777-785, doi: 10.1109/JSTSP.2009.2028380. [Link]

Smith, L., M. Richardson, and R. Walmsley, 2013: Comparison of MODTRAN5 to measured data in the UV band. Proc. SPIE 8898, Technologies for Optical Countermeasures X; and High-Power Lasers 2013: Technology and Systems, 88980F, doi: 10.1117/12.2028148. [Link]

Uudus, B., K. A. Park, K. R. Kim, J. Kim, and J. H. Ryu, 2013: Diurnal variation of NDVI from an unprecedented high-resolution geostationary ocean colour satellite. Remote Sens. Lett., 4, 639-647, doi: 10.1080/2150704X.2013.781285. [Link]

Wang, T. J., W. Z. Xu, and H. Lu, F. F. Ren, D. J. Chen, R. Zhang, and Y. D. Zheng, 2014a: Solar-blind ultraviolet band-pass filter based on metal-dielectric multilayer structures. Chin. Phys. B, 23, doi: 10.1088/16741056/23/7/074201. [Link]

Wang, X., Q. Zhang, and H. Li, 2014b: An autonomous navigation scheme based on starlight, geomagnetic and gyros with information fusion for small satellites. Acta Astronautica, 94, 708-717, doi: 10.1016/j.actaastro.2013.09.004. [Link]

Xiong, K., L. D. Liu, and H. Y. Zhang, 2009: Modified unscented Kalman filtering and its application in autonomous satellite navigation. Aero. Sci. Tech., 13, 238246, doi: 10.1016/j.ast.2009.04.001. [Link]

Xiong, K., C. Wei, and L. Liu, 2013: Autonomous navigation for a group of satellites with star sensors and inter-satellite links. Acta Astronautica, 86, 10-23, doi: 10.1016/j.actaastro.2012.12.001. [Link] 